\title{
Variations in shape among observed Lyman- $\alpha$ spectra due to intergalactic absorption
}

\author{
C. Byrohl ${ }^{1}$ and M. Gronke ${ }^{2,3, \star}$ \\ 1 Max-Planck-Institut für Astrophysik, Karl-Schwarzschild-Str. 1, 85748 Garching, Germany \\ e-mail: cbyrohl@mpa-garching.mpg.de \\ 2 Department of Physics and Astronomy, Johns Hopkins University, Baltimore, MD 21218, USA \\ 3 Department of Physics and Astronomy, University of California, Santa Barbara, CA 93106, USA
}

Received 18 June 2020 / Accepted 4 September 2020

\section{ABSTRACT}

\begin{abstract}
Lyman- $\alpha(\operatorname{Ly} \alpha)$ spectra provide insights into the small-scale structure and kinematics of neutral hydrogen (HI) within galaxies as well as the ionization state of the intergalactic medium (IGM). The former defines the intrinsic spectrum of a galaxy, which, in turn, is modified by the latter. These two effects are degenerate. Using the IllustrisTNG100 simulation, we studied the impact of the IGM on Ly $\alpha$ spectral shapes between $z \sim 0$ and 5. We computed the distribution of the expected Ly $\alpha$ peaks and of the peak asymmetry for different intrinsic spectra, redshifts, and large-scale environments. We find that the averaged transmission curves that are commonly applied give a misleading perception of the observed spectral properties. We show that the distributions of peak counts and asymmetry can lift the degeneracy between the intrinsic spectrum and IGM absorption. For example, we expect a significant number of triplepeaked Ly $\alpha$ spectra (up to $30 \%$ at $z \sim 3$ ) if the galaxies' HI distribution become more porous at higher redshift, as predicted by cosmological simulations. We provide a public catalog of transmission curves for simulations and observations to allow for a more realistic IGM treatment in future studies.
\end{abstract}

Key words. radiative transfer - intergalactic medium - large-scale structure of Universe - galaxies: high-redshift

\section{Introduction}

The Lyman- $\alpha(\operatorname{Ly} \alpha)$ line is a promising astrophysical observable for the neutral hydrogen distribution, from the scale of parsecs in star-forming regions (e.g., Kunth et al. 1998; Yang et al. 2016) all the way to cosmological scales. As such, it is among the most powerful observables to constrain the cosmic neutral fraction during the Epoch of Reionization (e.g., Dijkstra 2014; Mason et al. 2019).

While Ly $\alpha$ observations allow us to tackle a wide range of astrophysical questions, probing different spatial scales is a challenge because of a possible degeneracy of the origin of spectral features in those observations. Ly $\alpha$ observables are shaped not only through the neutral hydrogen (HI) distribution and the kinematics internal to galaxies, that is, the interstellar medium (ISM), but also by HI residing in the circumgalactic medium (CGM; Steidel et al. 2011; Wisotzki et al. 2016) and the intergalactic medium (IGM).

At low redshift $(z \lesssim 1)$, the impact of the IGM is very limited due to low neutral hydrogen fractions, such that observations with the Hubble Space Telescope reveal the spectral features imprinted by the ISM and CGM only. Usually, Ly $\alpha$ spectra show very little flux at line-center $\left(\lambda_{c} \sim 1216 \AA\right)$, and emission mostly originates on the red $\left(\lambda>\lambda_{c}\right)$ side of the spectrum, with a significant fraction of spectra showing essentially no flux on the blue $\left(\lambda<\lambda_{c}\right)$ side (Östlin et al. 2014; Hayes et al. 2014; Henry et al. 2015; Yang et al. 2016; review by Hayes 2015).

\footnotetext{
$\star$ Hubble fellow.
}

At higher redshifts, however, the impact of the IGM increases and the picture is less certain. Individual observations at $z \sim 5-7$ mainly show a single peak redshifted by a few hundred $\mathrm{km} \mathrm{s}^{-1}$ (Matthee et al. 2017). At intermediate redshifts, larger statistical samples do measure an asymmetry toward the red (Erb et al. 2014). This spectral evolution leaves, in principle, two possibilities: either the "intrinsic" Ly $\alpha$ spectra emergent from the galaxies do not vary strongly with redshift or they vary, but the IGM transmission, which is also evolving, makes the observed spectral properties remain similar. While the former scenario is supported by the fact that the low- $z$ samples are selected as "analogs" of higher redshift Ly $\alpha$ emitters (e.g., Yang et al. 2016), the latter is suggested by modern radiative transfer simulations using galactic hydrodynamical simulations as input (e.g., Laursen et al. 2011; Gronke et al. 2018; Smith et al. 2019). Due to strong feedback mechanisms, they produce a porous ISM at high- $z$, and thus, the predicted Ly $\alpha$ spectra exhibit relatively large flux at line-center and the blue side of the spectrum. Differentiating between these pathways is crucial to properly disentangle the Ly $\alpha$ line's use as a probe of galaxy and IGM evolution.

While recent studies have focused mainly on the intragalactic Ly $\alpha$ transfer (e.g., Smith et al. 2019), less attention has been devoted to the effect of the IGM on the Ly $\alpha$ spectral shape. Even large-scale studies that include the IGM have focused primarily on global statistics, such as the Ly $\alpha$ emitter clustering or luminosity function (e.g., Iliev et al. 2008; Zheng et al. 2010; Behrens et al. 2018; Byrohl et al. 2019). In this paper, we seek to clarify the IGM's impact on the Ly $\alpha$ spectra using using a recent cosmological simulation in the redshift range of $z=0-5$. 


\section{Methodology}

\subsection{Simulations}

We analyzed the IGM attenuation using the IllustrisTNG100 simulations (Naiman et al. 2018; Nelson et al. 2018; Marinacci et al. 2018; Pillepich et al. 2018; Springel et al. 2018) with a box size of 106.5 comoving Mpc for redshifts $0.0,1.0,2.0,3.0$, 4.0, and 5.0. The attenuation of Ly $\alpha$ flux was calculated with a modified version of ILTIS ${ }^{1}$, a line emission transfer code as per Behrens et al. (2019), which traces the optical depth in the IGM between the Ly $\alpha$ emitting galaxies and the observer for chosen lines of sight. The code was modified to natively run on IllustrisTNG's Voronoi tessellation. The initial tessellation was created with a parallelized wrapper of voro++ (Rycroft 2009) on IllustrisTNG's particle distribution. IllustrisTNG uses a time variable UV background with self-shielding (Faucher-Giguère et al. 2009; Rahmati et al. 2013) as well as a mimicking of the effect of active galactic nuclei (AGN) on the local radiation field (Vogelsberger et al. 2013) that is responsible for the hydrogen's ionization state in the IGM. Due to the long mean-free path of ionizing photons at the considered redshifts $(z \leq 5)$ and our exclusion of HI close to star-forming regions (see below), using a full radiation hydrodynamics simulation as input would not change the outcome of our study.

The optical depth, $\tau$, is integrated over the intervening medium for given lines of sight and a given input wavelength, $\lambda_{\mathrm{i}}$. The optical depth along the way can be expressed by

$\tau\left(\lambda_{\mathrm{i}}\right)=\int_{s_{0}}^{\infty} \mathrm{d} s n_{\mathrm{HI}}(s) \sigma\left(\lambda\left(\lambda_{\mathrm{i}}, v, s\right), T_{\mathrm{HI}}\right)$,

where $n_{\mathrm{HI}}$ is the neutral hydrogen density and $\sigma(\lambda)$ is the Ly $\alpha$ cross-section. In Eq. (1), we integrate over the physical distance, $s$, from the source along the chosen lines of sight (LOS). The temperature, $T_{\mathrm{HI}}$, sets the thermal broadening reshaping the cross-section profile $\sigma$. The cross-section is evaluated in the restframe of the gas and, thus, depends on the peculiar velocity $v$ and Hubble flow $H(z)$ at redshift $z$. The wavelength is shifted as $\lambda=\lambda_{\mathrm{i}}\left[1+\frac{v(s)+H(z) \cdot s}{c}\right]$, where $c$ is the speed of light. We commonly express the wavelength as its offset $\Delta \lambda_{e}=\lambda-\lambda_{c}$ from the Ly $\alpha$ line-center at the emitters' redshift.

The input wavelengths $\lambda_{\mathrm{i}}$ are evaluated in the rest-frame of the halos, which we identify as the mass-weighted velocity. We compute the optical depth within the wavelength range $\left[\lambda_{c}-5 \AA, \lambda_{c}+3 \AA\right]$ with a resolution of $0.02 \AA(R \sim 60000)$. For the IGM attenuation, we start summing contributions to the optical depth from a distance $s_{0}=f r_{\text {vir }}$, where $r_{\text {vir }}$ the virial radius of the halo. For a comparison with Laursen et al. (2011), we chose $f=1.5$. This implies that while photons are redshifted by the Hubble flow, no contributions to the optical depth are considered for $r<s_{0}$. The robustness of the chosen $s_{0}$ is confirmed in Appendix B. We integrate to $30 \mathrm{cMpch}^{-1}$ using periodic boundary conditions for the box. This length corresponds to a Hubble shift of $2600 \mathrm{~km} \mathrm{~s}^{-1}$ (at $z=1.0$ ) or more. We verified that for the chosen input wavelength range $\lambda_{\mathrm{i}}$, all wavelengths have significantly redshifted beyond the Ly $\alpha$ line-center as facilitated by the Hubble flow and, thus, no attenuation contributions are expected beyond this upper integration limit.

In our analysis, we consider the centers of halos as possible Ly $\alpha$-emitting galaxies if they contain regions of active star formation and have a total halo mass above $5 \cdot 10^{9} M_{\odot}$, as provided by IllustrisTNG's friends-of-friends halo catalogs. For

\footnotetext{
1 https://github.com/cbehren/Iltis
}

each emitter, we evaluate the optical depth along 1000 LOS. The LOS are constructed as normal vectors of a 1000-faced Fibonacci sphere, evenly tracing possible directions with the same normal vectors used throughout. A reduced public data set of our transmission curves has been published as Byrohl \& Gronke (2020) and a full data set can be made available upon request.

\subsection{Input spectra}

To demonstrate how the IGM attenuation affects the observed spectra in a statistical sample using the individual attenuation curves, we need to assume some input (intrinsic) spectra with flux density $I_{\lambda \text {,input }}$. Here, we use three different toy models:

First, we have a symmetric double peaked profile as result of a static neutral hydrogen sphere, also referred to as Neufeld solution (Neufeld 1990; Dijkstra et al. 2006). We set the temperature as $T_{\mathrm{HI}}=10^{4} \mathrm{~K}$ and the column density to $N_{\mathrm{HI}}=10^{20} \mathrm{~cm}^{-2}$ for this model.

Second, we consider red peak only that corresponds to the Neufeld solution under the assumption of a significant outflow. These two spectral shapes bracket the observed cases at low- $z$, which largely consist of a single or double peak dominant toward the red.

Third, we assume a Gaussian at Ly $\alpha$ line-center with a width of $\sigma=200 \mathrm{~km} \mathrm{~s}^{-1}$ as input spectrum. Such a setup with a significant line-center flux can be motivated for galaxies with a larger impact of stellar feedback at high redshifts leaving a more porous HI distribution. Ly $\alpha$ photons then escape closer to the line-center and are less susceptible to the gas kinematics (Neufeld 1991; Hansen \& Oh 2006; Gronke \& Dijkstra 2016). For this reason, recent galactic hydrodynamical models post-processed with Ly $\alpha$ radiative transfer show a wide, fairly symmetric intrinsic profile with little absorption at line-center emergent from these galaxies (e.g., Smith et al. 2019). The width was chosen to approximately match the predictions of those models.

\section{Results}

\subsection{Averaged transmission curves}

In Fig. 1, we show the resulting spectrum along three LOS for the same origin with the Gaussian and double-peaked input spectra. In this plot, and in general, it is mostly the spectrum blueward of the line-center that is affected as those frequencies will eventually shift into the line-center by the Hubble flow. Figure 1 shows that the transmission blueward of the line-center can fluctuate strongly for different wavelengths of a given line of sight. In fact, the LOS in Fig. 1 have been chosen such that the blue side of the observed spectrum exhibits a varying count of spectral peaks between zero and two for the Neufeld input spectrum. We formalize the count of peaks into a quantitative measure in Sect. 3.2.1. The transmission function is given as

$T\left(\Delta \lambda_{e}\right)=\exp \left[-\tau\left(\Delta \lambda_{e}\right)\right]$

and describes the fraction of the overall flux attenuated by the IGM between the emitter and the observer.

In Fig. 2, we show the probability density function (PDF) $p\left(T, \Delta \lambda_{e}\right)$ of the transmission function $T$ averaged over all emitters and LOS at $z=3$. We also plot the mean and median curves (blue/green bold lines) along with the central 68 percentiles (hatched area). From these curves, it is clear that the blue side is suppressed by roughly a factor of two, while the red side is mostly unaffected. We find a trough around line-center 


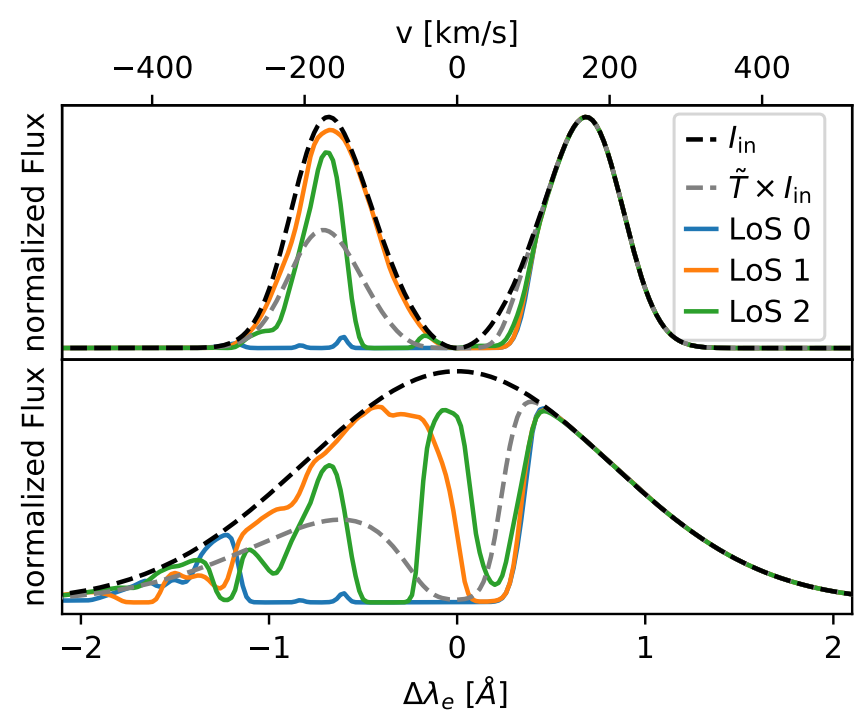

Fig. 1. Panels showing two different input (i.e., intrinsic) spectra (black lines) and how the IGM attenuation shapes the observed spectra along different LOS at $z=3.0$. All spectra are normalized by the respective underlying input spectrum's total flux. The gray dashed line shows the multiplication of the respective input spectrum with the median transmission curve, $\tilde{T}$, for all emitters and LOS at the given redshift. The colored solid lines show observed spectra for the same emitter but for different LOS. Wavelengths are evaluated in the halo's rest-frame. Top: input spectrum obtained as analytic solution of a spherical hydrogen distribution with a column density $N=10^{20} \mathrm{~cm}^{-2}$ and temperature $T=10^{4} \mathrm{~K}$ (Dijkstra et al. 2006). Bottom: with a Gaussian intrinsic spectrum with standard deviation $\sigma=200 \mathrm{~km} \mathrm{~s}^{-1}$.

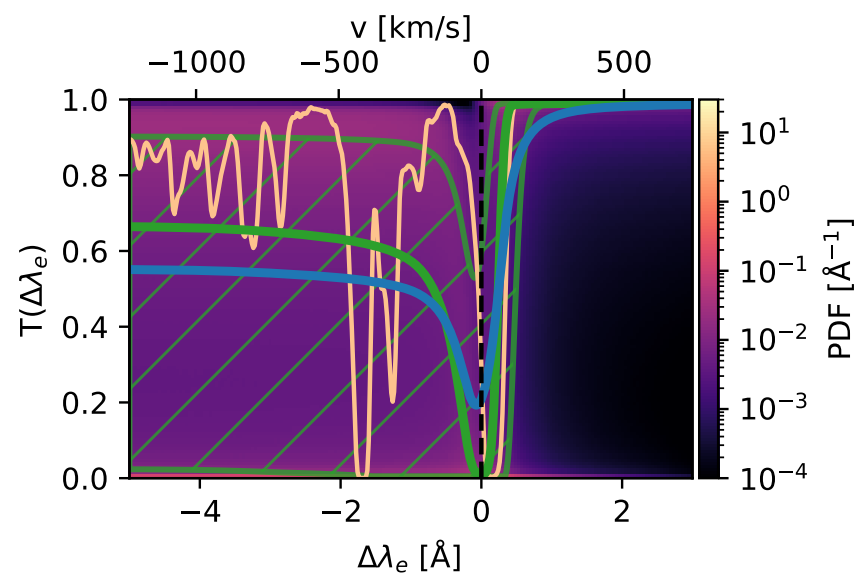

Fig. 2. PDF of the transmission, $T$, as a function input wavelength shift, $\Delta \lambda_{e}$, over all emitters and the LOS at $z=3$. Green line shows the median transmission with the hatched region enclosing the central $68 \%$ of all individual transmission curves, while the blue line shows the mean transmission curve over this PDF. The light orange curve shows an example of an individual LOS (corresponding to "LOS 1" in Fig. 1).

suppressing most of the flux. In general, we find these curves to be consistent with the results found by Laursen et al. (2011) and Gurung-López et al. (2020). Discrepancies in the asymptotic median value and the shaded 16th-84th percentile region are mostly a result of these quantities being highly dependent on the spectral resolution (see Appendix A). The spectral resolution in the literature is significantly lower with $R \lesssim 3000$ than $R \sim 60000$ used here.

For other redshifts, we find a similar qualitative trend over the shown wavelength range: the transmission on the blue side is increasingly suppressed at higher redshifts with a smaller asymptotic value toward $\Delta \lambda_{e}=-5 \AA$ and a deeper trough around $\Delta \lambda_{e}=0 \AA$. We find the most likely offset for this central trough to be roughly $20 \mathrm{~km} \mathrm{~s}^{-1}$ at $z=0.0$, monotonically increasing toward $70 \mathrm{~km} \mathrm{~s}^{-1}$ at $z=5.0$. These velocity offsets and their redshift evolution are consistent with expected halo infall velocities at $r=1.5 r_{\text {vir }}$ (Barkana 2004).

As illustrated by Figs. 1 and 2, overall, the median curve is misleading and should be interpreted with caution: the underlying PDF $p\left(T \mid \Delta \lambda_{e}\right)$ of transmission $T\left(\Delta \lambda_{e}\right)$ is usually bimodal, that is, due to the large Ly $\alpha$ cross-section, it is mostly close to zero or unity - which is not well-represented by averaged transmission curves. For instance, in Fig. 2, the bimodal distribution peaks around 0.0/0.9 (0.0/1.0) on the blue (red) side at $z=3.0$. These features can be seen more clearly in Appendix C.

While this bimodality becomes more pronounced at lower redshifts, a unimodal distribution with $\left\langle T\left(\Delta \lambda_{e}\right)\right\rangle \sim 0$ forms at higher redshifts as the upper bimodal transmission peak disappears. This bimodal behavior has significant consequences for the observed spectra. Rather than blue peaks being uniformly suppressed along different LOS, some LOS will show a strong blue feature while others will show none. Similarly there also is some variation for red peaks close to the line-center being suppressed given the bimodality there. In Sect. 3.2, we investigate two different quantitative measures to characterize the spectral variations for different lines of sight as implied here.

\subsection{Variations in transmission curves}

After studying the averaged transmission curves, we proceed to quantify the variations of the transmission curves. Those variations are observable features of the emerging spectra after traversing the IGM.

\subsubsection{Peak distribution}

As discussed in Sects. 1 and 2.2, the observed Ly $\alpha$ spectra at a low $z$ usually exhibit a double or single red peaked spectrum. Attenuation in the IGM can modify the observed peak count in some LOS.

For an observed spectral flux density $I_{\lambda}(\Delta \lambda)$, we define a peak as connected flux density bins such that $I_{\lambda}(\Delta \lambda)>I_{T}$ for a threshold $I_{T}$. Here, we set $I_{T}=0.01 \cdot \max _{0<\lambda<\infty}\left(I_{\lambda \text {,input }}\right)$. This criterion (while not its specific value) seeks to represent the flux sensitivity of a generic instrument. Furthermore, we require distinct connected areas to have a minimal separation of $0.2 \AA$ $(R \sim 6000)$ from one another. This criterion is aimed at avoiding any false identification of multiple peaks due to very small flux discontinuities that might additionally be below the spectral resolution of the spectrograph.

Figure 3 shows the distribution of the spectral peak count $0 \leq N_{\text {peaks }} \leq 3$ over the redshift range from 0 to 5 for the different input spectra. Hardly any LOS exist with $N_{\text {peaks }}>3$. The evolution with redshift is anchored by the intrinsic value of $N_{\text {peaks }}$ at $z \sim 0$ (i.e., $N_{\text {peaks }}=2$ and 1 for the double peaked input spectrum and the other two, respectively) and a single red peak at $z=5$, while in roughly $7 \%$ of all LOS, nearly all flux, and thus all peaks, are suppressed at $z=5$. For the intermediate redshifts, the rugged transmission curve (cf. Sect. 3.1) causes small attenuation features that substantially increase the number of observable peaks for the wide, central input spectrum. Specifically, the number of resultant double and even triple peaks increases to $\sim 50 \%$ and $30 \%$ at $z \sim 2-4$, respectively. Given that at higher redshifts, attenuation features become wider and only the most 


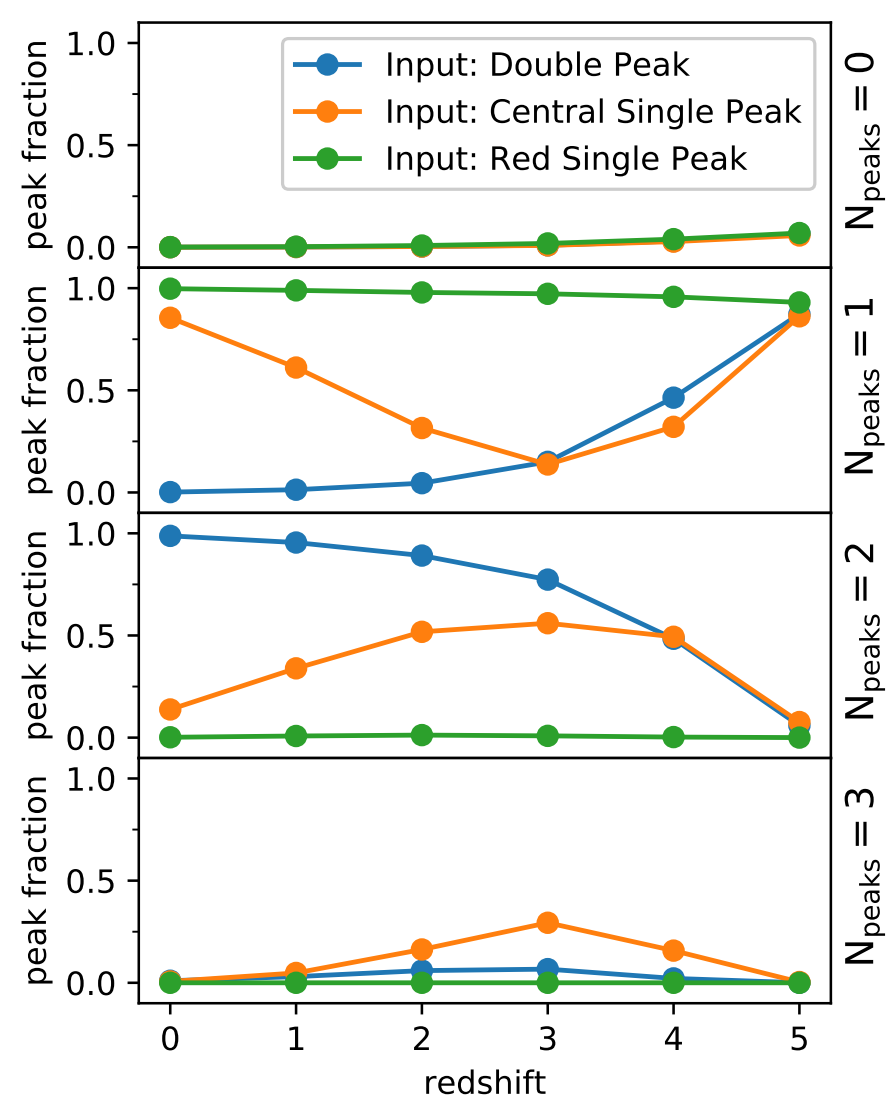

Fig. 3. Evolution of detectable Ly $\alpha$ peaks assuming the three different intrinsic spectra as described in Sect. 2.2. The peak detection algorithm is described in Sect. 3.2.1.

redward peak survives, this gives a typical U-shaped evolution for $N_{\text {peaks }} 1$ to 3 in this case. For the same reason, there is a slight U-shape in the triple peak count of the double peaked input spectrum. As the triple peak count is significantly at intermediate redshift, such a count is crucial in differentiating possible scenarios for the small-scale input spectra.

\subsubsection{Blue peak flux}

We introduce two observables to quantify the peak asymmetry. Namely, the flux ratio $L_{\text {ratio }}$ between the integrated flux $L_{\text {blue }}$ for wavelengths below the line-center and the total observed flux $\left(L_{\text {blue }}+L_{\text {red }}\right)$ :

$L_{\text {ratio }} \equiv \frac{L_{\text {blue }}}{L_{\text {blue }}+L_{\text {red }}}=\frac{\int_{0}^{\lambda_{c}} T(\lambda) \cdot I(\lambda) d \lambda}{\int_{0}^{\infty} T(\lambda) \cdot I(\lambda) d \lambda}$.

Analogously, we define the peak flux ratio $F_{\text {ratio }}$ as the ratio of maximal flux blueward to the sum of the peak fluxes on both sides of the line-center, namely:

$$
F_{\text {ratio }} \equiv \frac{F_{\text {blue }}}{F_{\text {blue }}+F_{\text {red }}}=\frac{\max _{0<\lambda<\lambda_{c}}(T(\lambda) \cdot I)}{\max _{0<\lambda<\lambda_{c}}(T(\lambda) \cdot I)+\max _{\lambda_{c}<\lambda<\infty}(T(\lambda) \cdot I)} .
$$

We note that observational studies have used similar measures in the past (e.g., Erb et al. 2014; Verhamme et al. 2017).

In Fig. 4, we show the distributions of $L_{\text {ratio }}$ and $F_{\text {ratio }}$ across all LOS (with both directions and emitters) using the double peaked intrinsic spectrum introduced in Sect. 2.2. The distribution looks very similar when using the Gaussian input spectrum.

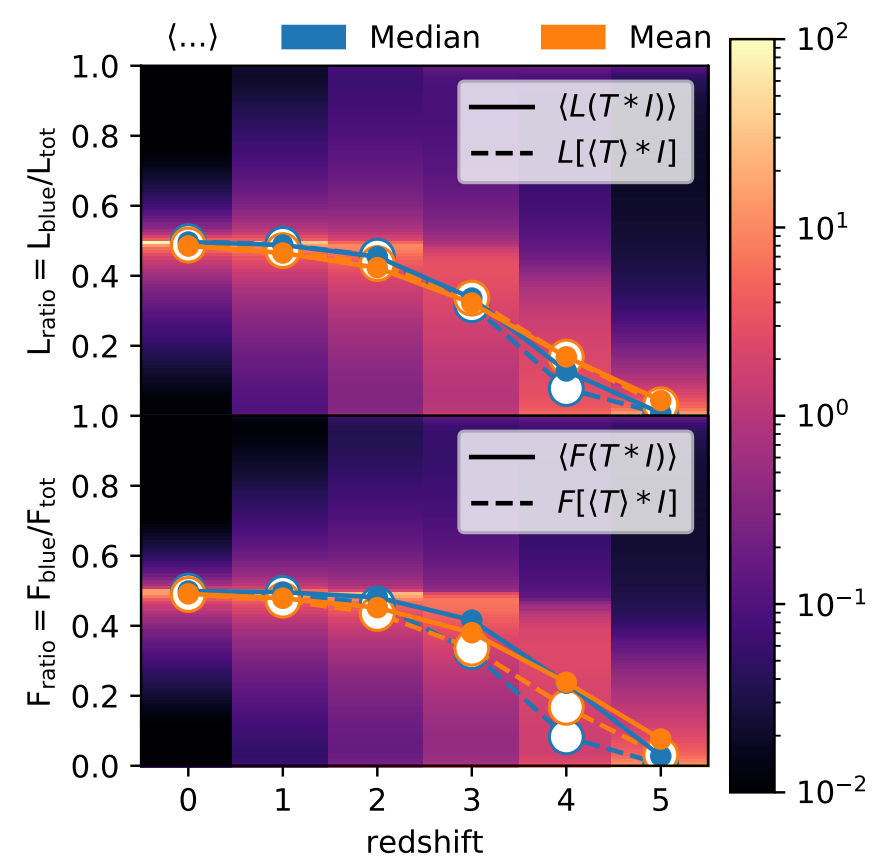

Fig. 4. Ratios $L_{\text {ratio }}$ (top) and $F_{\text {ratio }}$ (bottom) shown as a PDF over all lines of sight for both all directions and emitters at a given redshift as shown by the colormap. We chose the double peaked input spectrum, but we also find a very similar result for the centrally peaked input spectrum. The four overplotted lines in each panel show different averaging methods (see text). We assume that no peak can be detected (hence no ratio) when the flux remaining after passing the IGM is less than $1 \%$ of the intrinsic flux $I(\lambda)$.

We note that, in addition, we imposed a minimum flux on each side of the line-center of $1 \%$ the flux of the input spectrum for a LOS to be deemed detectable. A ratio of 0.5 signifies an equal impact of the IGM on the blue and red side of the line-center. Thus, while Fig. 4 makes a prediction for the observed distribution given the idealized input spectrum, it also represents the IGM's impact on the asymmetry as such, indicating a favorable escape of blue (red) photons through the IGM for values over (below) 0.5 .

The solid lines show the mean and the median for the ratios, while the dashed lines show ratio for the mean and median transmission curves $\langle T(\Delta \lambda)\rangle$ multiplied by the input spectra. Both ratios intuitively follow the expected redshift evolution for all lines: At redshift $z=0$, the asymmetry is mostly unaffected by the IGM, while at higher redshifts the averaged ratios drop toward zero at $z=5$ as the IGM becomes more opaque due to a higher HI density. A closer look at the peak asymmetry distribution at a given redshift reveals a more nuanced picture: At low to intermediate redshifts $(z \lesssim 3)$, the distribution appears somewhat symmetric around the median with increasing variance and skewness for higher redshifts. Therefore, blue peaks can be prominent features of a fraction of observed spectra even at high redshifts. We find fractions of $13.5 \%, 17.5 \%, 14.3 \%$, $8.5 \%, 4.1 \%$, and $1.8 \%$ from redshift 0 to 5 , enhancing the blue side of the spectrum (i.e., $L_{\text {ratio }}>0.5$ ). Observational studies calculating the enhanced blue peak fraction exist (Erb et al. 2014; Trainor et al. 2015) with up to $16-22 \%$ at $z \sim 3$. However, a fair comparison is not possible due to small number statistics, a varying definition of the flux ratio, and the very low spectral resolution. Future studies overcoming these challenges would be useful for constraining the (a)symmetry of the small-scale spectra. 

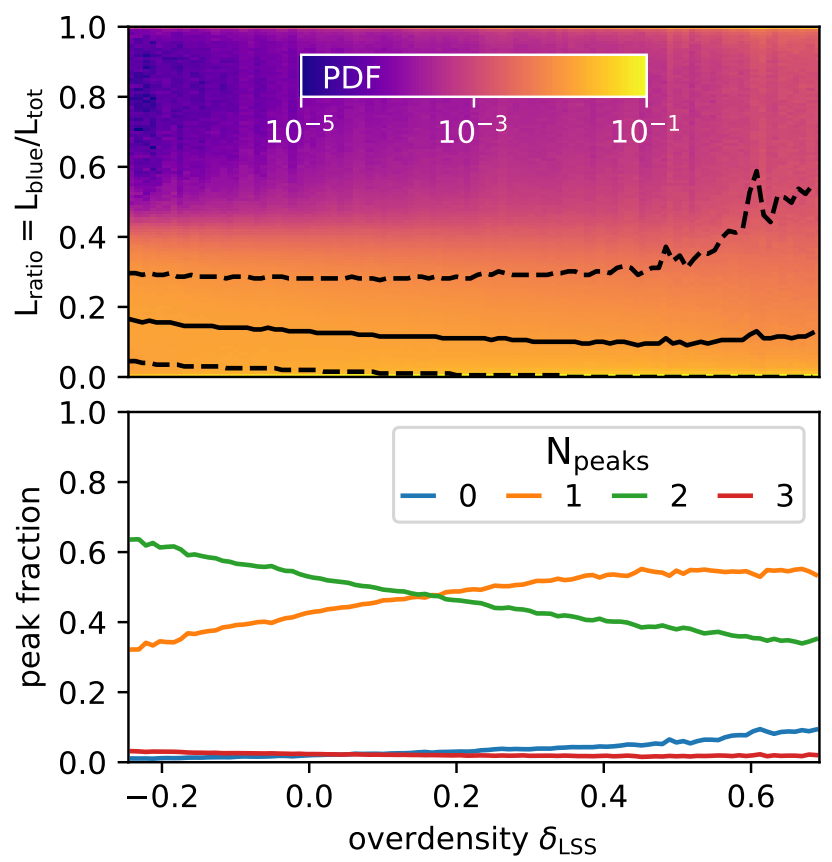

Fig. 5. $L_{\text {ratio }}$ and $N_{\text {peaks }}$ statistics shown as a function of the large scale overdensity $\delta_{\text {LSS }}$ at redshift $z=4.0$. The input spectrum is the Neufeld solution. The colormap in the upper panel shows the underlying PDF normalized at a given $\delta_{\text {LSS }}$. Overdense regions overall decrease $L_{\text {ratio }}$. At a higher overdensity, this trend halts as the ratio becomes more fluctuating for different LOS. For the peak fractions, we find a gradual decrease of double peaks. This is caused by absorption features on the blue side of the line-center. Thus, the decrease of double peaks is strongly correlated with the increase of the fraction of single peaked spectra.

A range of spectra will continue to contain significant blue contributions at high redshifts. This is another reason why the use of the averaged transmission curves could be misleading with regard to the underlying peak asymmetry distribution. For instance, for $z=5.0$, the median transmission curve leads to a $L_{\text {ratio }}$ on sub-percent level, leading to the perception that blue peaks are singularities at such redshift, when in reality we find roughly $4 \%$ of Ly $\alpha$ emitting galaxies that still show significant blue flux ( $L_{\text {blue }} \geq 0.25 \cdot L_{\text {red }}$; analogously, for $F$, we even find $10 \%)$. For the other redshifts $(0,1,2,3$, and 4$)$ we find $99.5 \%$, $97.0 \%, 91.8 \%, 75.5 \%$, and $33.3 \%$ of LOS with significant blue flux (if present intrinsically).

\subsubsection{Large-scale environment}

Beside the redshift and input spectrum, we find that our proposed statistics also depend on the emitters' large-scale environment. Most prominently, we find a correlation of the flux ratio and peak fraction with the linear overdensity as shown in Fig. 5 for $z=4.0$. We calculate the overdensity using a Gaussian smoothing kernel with $\sigma=3 \mathrm{cMpch}^{-1}$. The flux ratio slightly decreases toward higher overdensities indicating more intervening HI. Additionally, the scatter of the flux ratio strongly increases as more varying matter structure is passed along the LOS. The fraction of double peaks, as present in the input spectrum, strongly decreases toward higher overdensities as more blue peaks are attenuated. In up to $10 \%$ of the cases, the red peak is additionally suppressed, completely obscuring those Ly $\alpha$ emitting galaxies in high overdensity environments. This also affects the clustering signal of $\operatorname{Ly} \alpha$ emitters, as has been studied by Zheng et al. (2011) and Behrens et al. (2018) in more detail. The correlation with overdensity is much weaker for redshift between $z=0.0$ and 2.0, where the most likely outcome remains $L_{\text {ratio }}=0.5$ over the overdensity range. This behavior changes for $z=3.0$ and $z=4.0$ in overdense regions, where the distribution starts to get skewed toward $L_{\text {ratio }}$ close to zero. At higher redshifts, the correlation appears to be smaller as most blue peaks are already attenuated even in underdense regions.

\section{Conclusions}

The Ly $\alpha$ line can be used to constrain the neutral hydrogen distribution from galactic to cosmological scales. This versatility is, however, also a problematic since degeneracies between these scales exist that come into play at $z \gtrsim 3$, when the more opaque IGM can compensate the effects of a more porous ISM. Such a scenario - which is suggested by cosmological simulations allows not only for the escape of $\operatorname{Ly} \alpha$ photons closer to linecenter but also the escape of ionizing photons that are susceptible to the same galactic HI distribution (e.g., Dijkstra et al. 2016).

Using a large set of transmission curves that we calculated from the IllustrisTNG100 simulations, we quantified the impact of the IGM on spectra for $z=0-5$. In doing so, we studied a new approach to breaking the degeneracy using two statistics, namely the peak count and peak asymmetry (Sects. 3.2.1/3.2.2). In particular, we found the fraction of triple peaks to be an important differentiator for different intrinsic spectra. In contrast, we show that the commonly used averaged transmission curves can be misleading with regard to the interpretation of the impact of the IGM on observed Ly $\alpha$ spectra and their redshift evolution.

Making our catalogs of transmission curves publicly available (Byrohl \& Gronke 2020) opens this work up to other studies that look to incorporate an improved approach to IGM treatment and its impact on $\operatorname{Ly} \alpha$ spectra. At the same time, it can provide flexibility when refining the statistics presented and the intrinsic spectral modeling in the future.

Our findings require a high spectral resolution (optimally $R \gtrsim 6000$ ), particularly for the peak count statistic. Hence, current samples of Ly $\alpha$ spectra at $z \gtrsim 3$ have too low a spectral resolution for testing our predictions (e.g., Erb et al. 2014; Herenz et al. 2017). However, individual triple-peaked spectra have been observed (e.g., Rivera-Thorsen et al. 2017; Vanzella et al. 2020) and future surveys will be able to increase this count to a statistical sample that allows for the above-described degeneracy to be broken.

Acknowledgements. We thank Christoph Behrens for providing us with the early version of ILTIS, Eiichiro Komatsu and Shun Saito for useful discussions on this draft, and Dylan Nelson for his help processing the IllustrisTNG data. The radiative transfer simulations and analysis were conducted on the supercomputers at the Max Planck Computing and Data Facility (MPCDF). We acknowledge use of the Python programming language (Van Rossum \& de Boer 1991), and use of the Astropy (Astropy Collaboration 2013), Numpy (van der Walt et al. 2011), IPython (Perez \& Granger 2007), Dask (Dask Development Team 2016), h5py (Collette 2013) and Matplotlib (Hunter 2007) packages for post-processing. M. G. was supported by NASA through the NASA Hubble Fellowship grant HSTHF2-51409 and acknowledges support from HST grants HST-GO-15643.017-A, HST-AR-15039.003-A, and XSEDE grant TG-AST180036.

\section{References}

Astropy Collaboration (Robitaille, T. P., et al.) 2013, A\&A, 558, A33

Barkana, R. 2004, MNRAS, 347, 59

Behrens, C., Byrohl, C., Saito, S., \& Niemeyer, J. C. 2018, A\&A, 614, A31

Behrens, C., Pallottini, A., Ferrara, A., Gallerani, S., \& Vallini, L. 2019, MNRAS, 486, 2197

Byrohl, C., \& Gronke, M. 2020, Lyman-alpha Transmission Curves (Zenodo) 
Byrohl, C., Saito, S., \& Behrens, C. 2019, MNRAS, 489, 3472 Collette, A. 2013, Python and HDF5 (O'Reilly)

Dask Development Team 2016, Dask: Library for Dynamic Task Scheduling Dijkstra, M. 2014, PASA, 31, e040

Dijkstra, M., Haiman, Z., \& Spaans, M. 2006, ApJ, 649, 14

Dijkstra, M., Gronke, M., \& Venkatesan, A. 2016, ApJ, 828, 71

Erb, D. K., Steidel, C. C., Trainor, R. F., et al. 2014, ApJ, 795, 33

Faucher-Giguère, C.-A., Lidz, A., Zaldarriaga, M., \& Hernquist, L. 2009, ApJ, 703,1416

Gronke, M., \& Dijkstra, M. 2016, ApJ, 826, 14

Gronke, M., Girichidis, P., Naab, T., \& Walch, S. 2018, ApJ, 862, L7

Gurung-López, S., Orsi, Á. A., Bonoli, S., et al. 2020, MNRAS, 491, 3266

Hansen, M., \& Oh, S. P. 2006, MNRAS, 367, 979

Hayes, M. 2015, PASA, 32, e027

Hayes, M., Östlin, G., Duval, F., et al. 2014, ApJ, 782, 6

Henry, A., Scarlata, C., Martin, C. L., \& Erb, D. 2015, ApJ, 809, 19

Herenz, E. C., Urrutia, T., Wisotzki, L., et al. 2017, A\&A, 606, A12

Hunter, J. D. 2007, Comput. Sci. Eng., 9, 90

Iliev, I. T., Shapiro, P. R., McDonald, P., Mellema, G., \& Pen, U.-L. 2008, MNRAS, 391, 63

Kunth, D., Mas-Hesse, J. M., Terlevich, E., et al. 1998, A\&A, 334, 11

Laursen, P., Sommer-Larsen, J., \& Razoumov, A. O. 2011, ApJ, 728, 52

Marinacci, F., Vogelsberger, M., Pakmor, R., et al. 2018, MNRAS, 480, 5113

Mason, C. A., Fontana, A., Treu, T., et al. 2019, MNRAS, 485, 3947

Matthee, J., Sobral, D., Darvish, B., et al. 2017, MNRAS, 472, 772
Naiman, J. P., Pillepich, A., Springel, V., et al. 2018, MNRAS, 477, 1206 Nelson, D., Pillepich, A., Springel, V., et al. 2018, MNRAS, 475, 624 Neufeld, D. A. 1990, ApJ, 350, 216

Neufeld, D. A. 1991, ApJ, 370, L85

Östlin, G., Hayes, M., Duval, F., et al. 2014, ApJ, 797, 11

Perez, F., \& Granger, B. E. 2007, Comput. Sci. Eng., 9, 21

Pillepich, A., Nelson, D., Hernquist, L., et al. 2018, MNRAS, 475, 648

Rahmati, A., Pawlik, A. H., Raičević, M., \& Schaye, J. 2013, MNRAS, 430, 2427

Rivera-Thorsen, T. E., Dahle, H., Gronke, M., et al. 2017, A\&A, 608, L4

Rycroft, C. H. 2009, Chaos: Interdiscip. J. Nonlinear Sci., 19, 041111

Smith, A., Ma, X., Bromm, V., et al. 2019, MNRAS, 484, 39

Springel, V., Pakmor, R., Pillepich, A., et al. 2018, MNRAS, 475, 676

Steidel, C. C., Bogosavljević, M., Shapley, A. E., et al. 2011, ApJ, 736, 160 Trainor, R. F., Steidel, C. C., Strom, A. L., \& Rudie, G. C. 2015, ApJ, 809, 89 van der Walt, S., Colbert, S. C., \& Varoquaux, G. 2011, Comput. Sci. Eng., 13, 22

Van Rossum, G., \& de Boer, J. 1991, CWI Q., 4, 283

Vanzella, E., Caminha, G. B., Calura, F., et al. 2020, MNRAS, 491, 1093

Verhamme, A., Orlitová, I., Schaerer, D., et al. 2017, A\&A, 597, A13

Vogelsberger, M., Genel, S., Sijacki, D., et al. 2013, MNRAS, 436, 3031

Wisotzki, L., Bacon, R., Blaizot, J., et al. 2016, A\&A, 587, A98

Yang, H., Malhotra, S., Gronke, M., et al. 2016, ApJ, 820, 130

Zheng, Z., Cen, R., Trac, H., \& Miralda-Escudé, J. 2010, ApJ, 716, 574

Zheng, Z., Cen, R., Trac, H., \& Miralda-Escudé, J. 2011, ApJ, 726, 38 


\section{Appendix A: Spectral resolution}

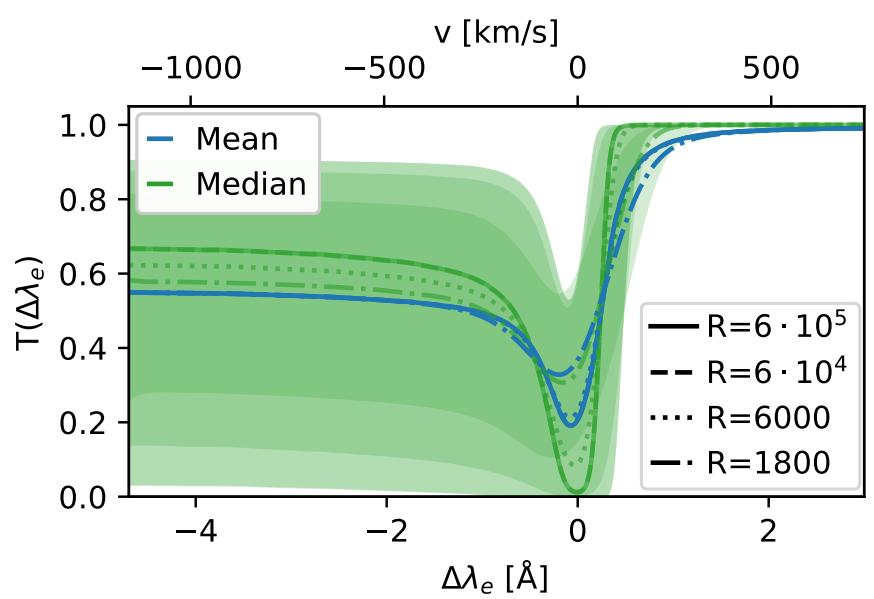

Fig. A.1. Impact of the spectral resolution on the PDFs of the transmission function $T$ as a function of input wavelength shift $\Delta \lambda_{e}$. We show the PDF of $T$ over all emitters and LOS at $z=3$, similarly to Fig. 2. In blue we show the mean for a given $\Delta \lambda_{e}$, while in green we show the median. We show the transmission at different spectral resolutions $R \in\{600000,60000,6000,1800\}$ (solid, dashed, dotted, dash-dotted). Resolutions below 600000 are computed as convolution with a Gaussian of FWHM $\lambda_{F W H M}$ that sets the spectral resolution as $R=\lambda_{c} / \lambda_{F W H M}$. The shaded regions enclose the central $68 \%$ of all individual transmission curves. The darkest (lightest) shade corresponds to the lowest (highest) spectral resolution. As the resolution increases the median increases and the shaded region widens. At our fiducial resolution of $\sim 60000$ these statistics have converged.

In Fig. A.1 we show the impact of the spectral resolution on the averaged transmission curve at $z=3$. We show the median along with the 16th and 84th percentile and the mean over the different emitters and LOS for at a given wavelength offsets $\Delta \lambda_{e}$. We find that the mean is nearly independent of the spectral resolution between 6000 and 600000 , while the median and percentiles in general strongly dependent on chosen resolution. We note that the result has converged at $R \sim 60000$, which is our fiducial resolution throughout this paper. At $R=1800$ the smoothing is larger than the size of the transmission curve's central trough and both mean and median thus significantly change in its proximity.

Our simulations at $R \sim 60000$ are expected to be converged due to the Doppler broadening in the line profile. The full width at half maximum (FWHM) due to Doppler broadening is given as $\lambda_{F W H M} \approx 2.35 \lambda_{c} \sqrt{\frac{k_{B} T}{m_{p} c^{2}}}$. Assuming an IGM temperature of $T \sim 10^{4} \mathrm{~K}$, this implies a spectral resolution of $R \sim 14000$. Features imprinted by the hydrogen structure on smaller scales will thus be washed out.

The difference in behavior of median and percentiles compared to the mean is easily explained: linearity, that is, STAT $[A+B]=$ STAT $[A]+\operatorname{STAT}[B]$ for two distributions, $A$ and $B$, and a summary statistic, STAT, holds for the mean but not for percentiles such as the median. Take, for example, two PDFs, $A=P(T)_{\Delta \lambda_{e}=\lambda_{i}}$ and $B=P(T)_{\Delta \lambda_{e}=\lambda_{i}}$, where $\lambda_{i}$ and $\lambda_{j}$ describe two neighboring wavelength bins. The mean transmissivity $\mathrm{T}$ over those two bins can be determined from the mean of $A$ and $B$ alone, while this is not possible for the median. In particular, if two neighboring bins have the same mean, the mean of the sum of those distributions has to be the same, whereas this is not true for the median, as can be readily seen in Fig. A.1. This behavior is furthermore complicated for the median as the distributions in neighboring bins are strongly correlated. To address this issue, we recommend using the mean transmission curves over the median unless the spectral resolution is clearly stated.

We obtain a triple peak fraction of $28.5 \%, 28.4 \%, 17.6 \%$, and $0.1 \%$ for spectral resolutions of $600000,60000,6000$, and 1800 given the Gaussian input spectrum. Thus, in addition, the peak count is converged at our fiducial resolution of 60000 . At typical resolutions of spectrographs today $(R \sim 1800)$, triple peaks are rare to non-existent for given small-scale spectrum.

\section{Appendix B: Start of integration}

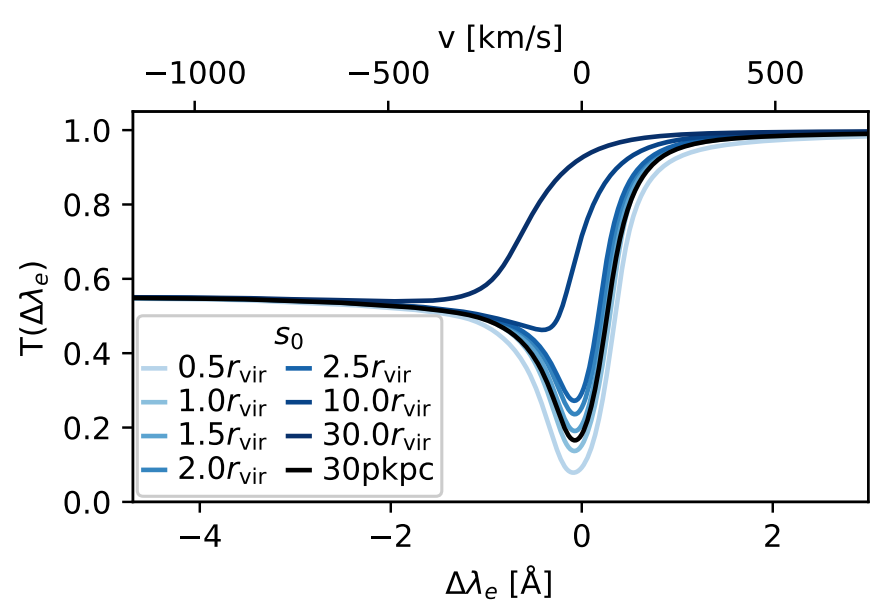

Fig. B.1. Impact of the lower integration bound $s_{0}$ for mean transmission curves. We averaged over all LOS and Ly $\alpha$ - emitting galaxy candidates at $z=3$. We show the cases of $f=0.5,1.0,2.0,2.5$ around our fiducial case of 1.5. These results are contrasted with cases $f=10.0,30.0$ for interaction on large scales only. In addition, we show the case of a fixed $s_{0}=30 \mathrm{pkpc}$ irrespective of virial radius of the underlying halo.

In Fig. B.1, we vary the start $s_{0}$ of the integral for the optical depth and show the resulting mean transmission curves. For small changes around $s_{0}=1.5 \pm 1.0 r_{\text {vir }}$, we find the central trough's depth to slightly change and becoming deeper for smaller values of $s_{0}$. Going beyond $f=10.0$ removes the relevant scale responsible for the mean attenuation curve's central trough. On an even larger scale $(f=30.0)$, attenuation is shifted toward lower input wavelengths due to the Hubble flow.

The triple peak count remains stable around $\sim 30 \%$ with $28.9 \%, 30.2 \%, 29.4 \%, 28.2 \%$, and $27.1 \%$ for $f$ equal to $0.5,1.0$, $1.5,2.0$, and 2.5 with $s_{0}=f r_{\text {vir }}$. Similarly, when using a constant $s_{0}$ rather than depending on the virial radius we obtain $29.6 \%$ for $s_{0}=30 \mathrm{pkpc}$. Thus, our results are robust for chosen $f=1.5$.

The peak fraction is largely determined in the proximity of the targeted halo. The triple peak fraction significantly drops to $17.6 \%$ and $6.1 \%$ for $f=10.0$ and $f=30.0$, showing that spectra are shaped both in the proximity of emitters $(f \gtrsim 2.5)$ and on large scales $(f \gtrsim 10.0)$. 


\section{Appendix C: Bimodality of the transmission curves}

In Fig. C.1, we show the distribution of transmission coefficients $T$ at fixed input wavelengths $\Delta \lambda_{e}$ at $z=3.0$. We show three different wavelengths: Blue of the line-center (blue line), in the line-center itself (green line), and red of the line-center (red line). The bimodality for the line-center and larger wavelengths is peaking at $T$ zero or unity, while shorter wavelengths show their second peak below unity ( $\sim 0.9$ here). This second peak's existence and position is redshift dependent (see main body). We also show the medians (arrows) color coded for the according distribution. For the line-center and redder wavelengths, one peak is commonly so dominant that the median is shifted to there accordingly. While in this case, the median is aligned with one of the peaks, this is not the case for blue wavelengths.

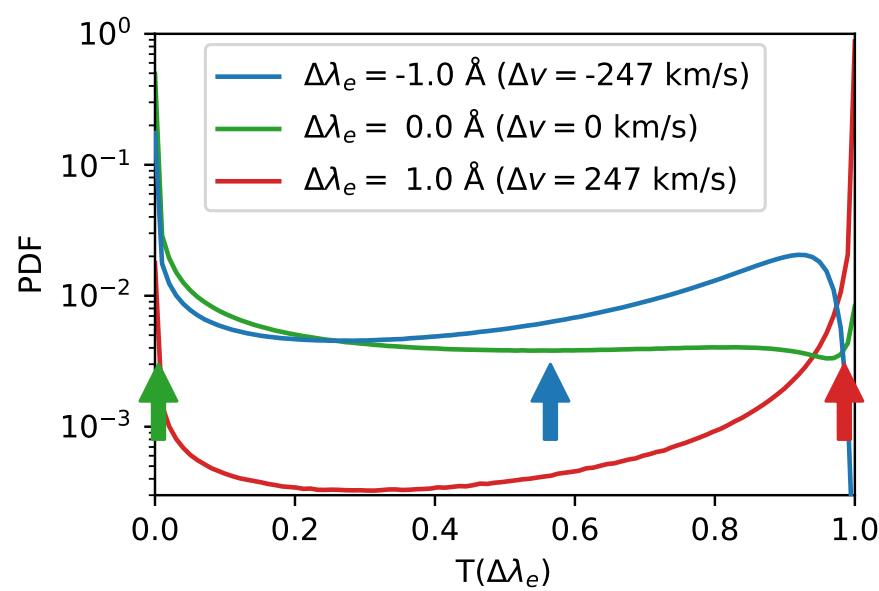

Fig. C.1. Distribution of transmission $T$ at fixed input wavelength offset $\Delta \lambda_{e}$ at $z=3.0$ across all emitters and LOS. The arrows show the accordingly color coded median. 